\title{
Expression and clinical significance of EGR-1 and PTEN in the pituitary tumors of elderly patients
}

\author{
SHU-WEN SUN, XIAO-MEI FANG, YI-FEI LI, QING-BO WANG and YU-XIN LI \\ Department of Neurosurgery, Health Examination Center, The Binhai Development Zone \\ People's Hospital of Weifang, Weifang, Shandong 262737, P.R. China
}

Received June 17, 2016; Accepted December 12, 2017

DOI: $10.3892 / \mathrm{ol} .2017 .6375$

\begin{abstract}
The aim of the study was to investigate the expression and clinical significance of early growth response protein 1 (EGR-1) and phosphatase and tensin homolog (PTEN) in the pituitary tumors of elderly patients. From January 2014 to December 2015, we collected 25 patient cases with non-invasive pituitary tumors, 10 cases with invasive pituitary tumors and 35 cases with healthy pituitary tissues (the healthy control group). Immunohistochemical staining was used to detect the expression of EGR-1 and PTEN, and analyze specific differences. The expression of EGR-1 and PTEN in patients with invasive and non-invasive pituitary tumors increased significantly, when compared with the healthy control group, and the difference was statistically significant $(\mathrm{p}<0.05)$. In patients with invasive tumors, EGR-1 levels were higher than levels in patients with non-invasive tumors. The difference was statistically significant $(\mathrm{p}<0.05)$. PTEN levels in patients with invasive tumors were significantly lower than levels in patients with non-invasive tumors. The difference was statistically significant $(\mathrm{p}<0.05)$. In conclusion, EGR-1 and PTEN levels in patients with pituitary tumors were significantly higher. In addition, EGR-1 levels were higher in patients with invasive pituitary tumors, while PTEN levels were lower. The combination of increases in both levels highlights an important role in the evaluation and prognosis of elderly patients with pituitary tumors.
\end{abstract}

\section{Introduction}

Pituitary tumors occur in the anterior and posterior pituitary of the pituitary gland and in the epithelium of the craniopharyngeal duct (1). Clinically, obvious symptoms account

Correspondence to: Dr Yu-Xin Li, Department of Neurosurgery, Health Examination Center, The Binhai Development Zone People's Hospital of Weifang, Binhai Economic Development Zone, 05441 Xihai Road, Weifang, Shandong 262737, P.R. China E-mail: jbn537519@163.com

Key words: early growth response protein 1, phosphatase and tensin homolog for approximately $10 \%$ of intracranial tumors. These tumors occur in males slightly more than females. Pituitary tumors usually occur in young adults and often affect growth and development, reproductive function, learning and the working ability of patients (2-4). Clinical manifestations include hormone secretion disorders, tumor compressions of the surrounding tissues of the pituitary gland, pituitary apoplexy and the decline of other anterior pituitary functions $(5,6)$. In recent years, with the rise of tumor markers, the study on pituitary tumors has gradually developed to the molecular level (7).

EGR-1, is an early growth response 1-encoded protein (8). It is believed that EGR-1 plays an important role in regulating proliferation, differentiation, apoptosis and embryonic development of early pituitary tissues and in the occurrence and development processes of a variety of benign and malignant tumors, including genital tumors and tumors of the digestive system (9). PTEN, is a phosphatase and tension homolog encoded protein (10). Previous findings showed that PTEN is one of the many new genes that inhibits the growth of the pituitary gland (11).

The present study used the immunohistochemical SP method to detect the protein expression in pituitary adenomas and, according to pathological staging, to observe different expression changes and examine the role and possible mechanism involved in the occurrence and development of pituitary tumors to provide a preliminary experimental basis for the prevention, early diagnosis and target gene treatment of the disease.

\section{Materials and methods}

Inclusion criteria and exclusion criteria. From January 2014 to September 2015, we collected 35 cases confirmed by pathological diagnosis as senile pituitary tumors from the hospital's Dermatology Clinic. These consisted of 25 cases of non-invasive pituitary adenomas and 10 cases of invasive pituitary adenomas. At the same time, 35 cases of normal pituitary tissues were taken from the patients. This experiment was approved by the University Medical Ethics Committee at the Binhai Development Zone People's Hospital (Weifang, China).

The inclusion criteria of the study were as follows: i) Senile pituitary tumors by pathological diagnosis; ii) patient had no other primary or secondary benign or malignant tumors; 
iii) patient was first diagnosed; and iv) patient voluntarily participated and signed an informed consent form.

The exclusion criteria of the study were: i) Patient and family members could not participate in the experiment; ii) patient refused to accept pathological examination; iii) patient refused treatment; iv) patient was treated with chemotherapy, radiotherapy, targeted therapy or immune regulation 3-4 weeks before he or she was had pathologic tissue cutting performed and was enrolled in this study; and v) patient had mental illness or a consciousness disorder.

Test reagent. The solutions used in the study were: $0.9 \%$ sterile saline (Otsuka Pharmaceutical Co., Ltd., Tokyo, Japan), mouse anti-human EGR-1 antibody and mouse anti-human PTEN antibody (both from Wuhan Boster Bio-Engineering Co., Ltd., Wuhan, China), immunohistochemical SP900 staining kit, diaminobenzidine (DAB) concentration staining kit and Mayer's hematoxylin (all from Zhongshan Jinqiao Biotechnology Co., Ltd., Beijing, China), fresh xylene (Tianjin Fu Chen Chemical Reagents Factory, Tianjin, China), $35 \%$ ethanol, $\mathrm{NaH}_{2} \mathrm{PO}_{4} 2 \cdot \mathrm{H}_{2} \mathrm{O}, \mathrm{Na}_{2} \mathrm{HPO}_{4} 12 \cdot \mathrm{H}_{2} \mathrm{O}$, citric acid and sodium citrate (all from Tianjin Fu Chen Chemical Reagents Factory, Tianjin, China), and water bath (Beijing Medical Equipment Factory, Beijing, China).

Experimental instruments. The instruments used in the present study were: Micropipettes (Eppendorf AG, Hamburg, Germany), refrigerator (set at $4^{\circ} \mathrm{C}$ ) (Haier Group, Shandong, China), 10 and $5 \mathrm{ml}$ syringe (both from Tianjin Hanahao Medical Materials Co., Ltd., Tianjin, China), pathological microtome (Leica Microsystems GmbH, Wetzlar, Germany), EP tube (Eppendorff AG), micro camera and optical microscope (both from Olympus, Tokyo, Japan).

Pathological drawing method. Licensed physicians removed skin tissue from suspected lesions. Prior to the incision, lidocaine was used for local anesthesia. Skin tissue samples were immediately placed in a container with $4 \%$ paraformaldehyde solution. The section was embedded by the Department of Pathology of the Binhai Development Zone People's Hospital. Using the method of serial section, the physicians set the thickness at $5 \mu \mathrm{m}$, and recorded the patient's name, medical record number, sampling time and sampling tissue types. The samples were placed into an oven at $60^{\circ} \mathrm{C}$ for $1 \mathrm{~h}$, to enable the tissue to closely attach to the slide. Samples were removed from the oven and cooled to room temperature.

Immunohistochemical SP method. Prior to immunohistochemical staining, the paraffin section of the pathological tissue was initially tested (12). The steps of paraffin section testing included fixation, dehydration, transparency, tissue embedding, slicing and using a baking sheet. After generating the paraffin section, the immunohistochemical SP method was used to perform histochemical staining.

The staining steps were as follows: i) Dewaxing: at $20^{\circ} \mathrm{C}$ and standing for $60 \mathrm{~min}$, soaked with xylene for $25 \mathrm{~min}$; ii) hydration: Soaked in anhydrous alcohol for 2 min before being placed in a solution of 95,80 and $70 \%$ alcohol, respectively, each for $2 \mathrm{~min}$; phosphate-buffered saline (PBS) rinse performed 2-3 times, each rinse for 5 min. iii) Blocking:
Use of $3 \% \mathrm{H}_{2} \mathrm{O}_{2}$ deionized water and incubation for $10 \mathrm{~min}$ followed by PBS rinsing 2-3 times, with each rinse for $5 \mathrm{~min}$. iv) Antigen repair: in $95^{\circ} \mathrm{C}$ citric acid buffer solution ( $\mathrm{pH} 6.0$ ) and heating for $15-20 \mathrm{~min}$, followed by cooling at room temperature for $20 \mathrm{~min}$; placed in cold water and then into a cylinder at room temperature; PBS rinse performed 2-3 times, each rinse for $5 \mathrm{~min}$. v) Enclosed: Use of normal goat serum blocking at room temperature and incubation for $20 \mathrm{~min}$, removal of excess liquid; use of primary antibody $(50 \mu 1)$, incubating at $20^{\circ} \mathrm{C}$ for $1 \mathrm{~h}$. PBS rinse was then peformed 2-3 times, with each rinse for $5 \mathrm{~min}$ followed by addition of 40-50 $\mu$ l horseradish peroxidase secondary monoclonal goat anti-rabbit IgG antibody (dilution, 1:2,000; cat. no. ab6721; Abcam, Cambridge, MA, USA), incubating at room temperature for $1 \mathrm{~h}$. PBS rinsing was then performed 2-3 times, with each rinse for $5 \mathrm{~min}$, followed by the addition of streptavidin peroxidase and incubation at room temperature for $30 \mathrm{~min}$ prior to PBS rinse 2-3 times, with each rinse for $5 \mathrm{~min}$. vi) Color: Samples were immersed into DAB color development solution for 5-10 min and viewed under the microscope to note the degree of staining. A brown cytoplasm was determined as the positive pituitary (PP) gland, and a tap water rinse was performed for $10 \mathrm{~min}$ to terminate the reaction. vii) Complex: Performed hematoxylin staining for $2 \mathrm{~min}$, followed by the addition of hydrochloric acid alcohol for differentiation prior to a tap water rinse of $10-15$ min. viii) Dehydrate, clean and mount: Neutral gum was used under the tissue, and this was covered with the coverslip for microscopic examination.

Interpretation method of immunohistochemical staining results of senile pituitary tumor. The results of the staining were interpreted and scored as follows: i) Positive detection: Product protein was found to be positive for EGR-1 and PTEN gene expression in the pituitary gland. After staining and under optical microscope, pituitary nuclear particles were viewed as brown or tan, indicating positive designation; ii) pituitary count: The pituitary count was expressed as $\mathrm{PP} / \mathrm{mm}^{2}$, this factor was scored according to the observed EGR-1 and PTEN $\mathrm{PP}$ ratio and staining intensity (SI); iii) scoring criteria: Total score $=$ SI $x$ PP. A total score of $\leq 6$ and $>6$ were defined as negative and positive, respectively. In accordance with the pituitary staining intensity, testing was divided into 4 levels: i) No pituitary staining was negative, 0 points; ii) weakly positive (+), 1 point; iii) moderately positive $(++), 2$ points; and iv) strongly positive $(+++), 3$ points.

According to the percentage of PP, testing was divided into four levels: i) Negative: No pituitary staining, 0 points; ii) positive: Pituitary $\leq 10 \%, 1$ point; iii) PP $11-50 \%, 2$ points; iv) $\mathrm{PP} 51-80 \%, 3$ points; and v) $\mathrm{PP}>80 \%, 4$ points. The positive rate of samples was calculated by the percentage of positive samples in the total number of samples. Ten randomly selected fields were viewed by high magnification, $\mathrm{x} 400$, calculated as a percentage of PP samples. Mean \pm SD was the average value of the PP and its standard deviation was the expression intensity.

Statistical analysis. SPSS 19.0 software (IBM SPSS, Armonk, NY, USA) was used for statistical analysis, and the qualitative data were analyzed and tested by analysis of variance (ANOVA) test, t-test and $\chi^{2}$ test. The Fisher's precise probability method was used for the 4 grid data of who did 
Table I. Comparison of the positive rates of EGR-1 and PTEN genes in tissue samples.

\begin{tabular}{|c|c|c|c|c|c|c|c|}
\hline $\begin{array}{l}\text { Genes for } \\
\text { detection }\end{array}$ & Groups & No. of cases & Positive & Negative & Positive rate $(\%)$ & $\chi^{2}$-value & P-value \\
\hline \multirow[t]{3}{*}{ EGR-1 } & Invasive pituitary tumor & 25 & 19 & 6 & $76.0(19 / 25)$ & 8.9 & 0.007 \\
\hline & Noninvasive pituitary tumor & 10 & 6 & 4 & $60.0(6 / 10)$ & & \\
\hline & Healthy pituitary tissue & 35 & 2 & 33 & $6.06(2 / 35)$ & & \\
\hline \multirow[t]{3}{*}{ PTEN } & Invasive pituitary tumor & 25 & 22 & 3 & $88.0(22 / 25)$ & 56.7 & $<0.0001$ \\
\hline & Noninvasive pituitary tumor & 10 & 7 & 3 & $70.0(7 / 10)$ & & \\
\hline & Healthy pituitary tumor tissue & 35 & 2 & 33 & $6.06(2 / 35)$ & & \\
\hline
\end{tabular}

EGR-1, early growth response protein 1; PTEN, phosphatase and tensin homolog.
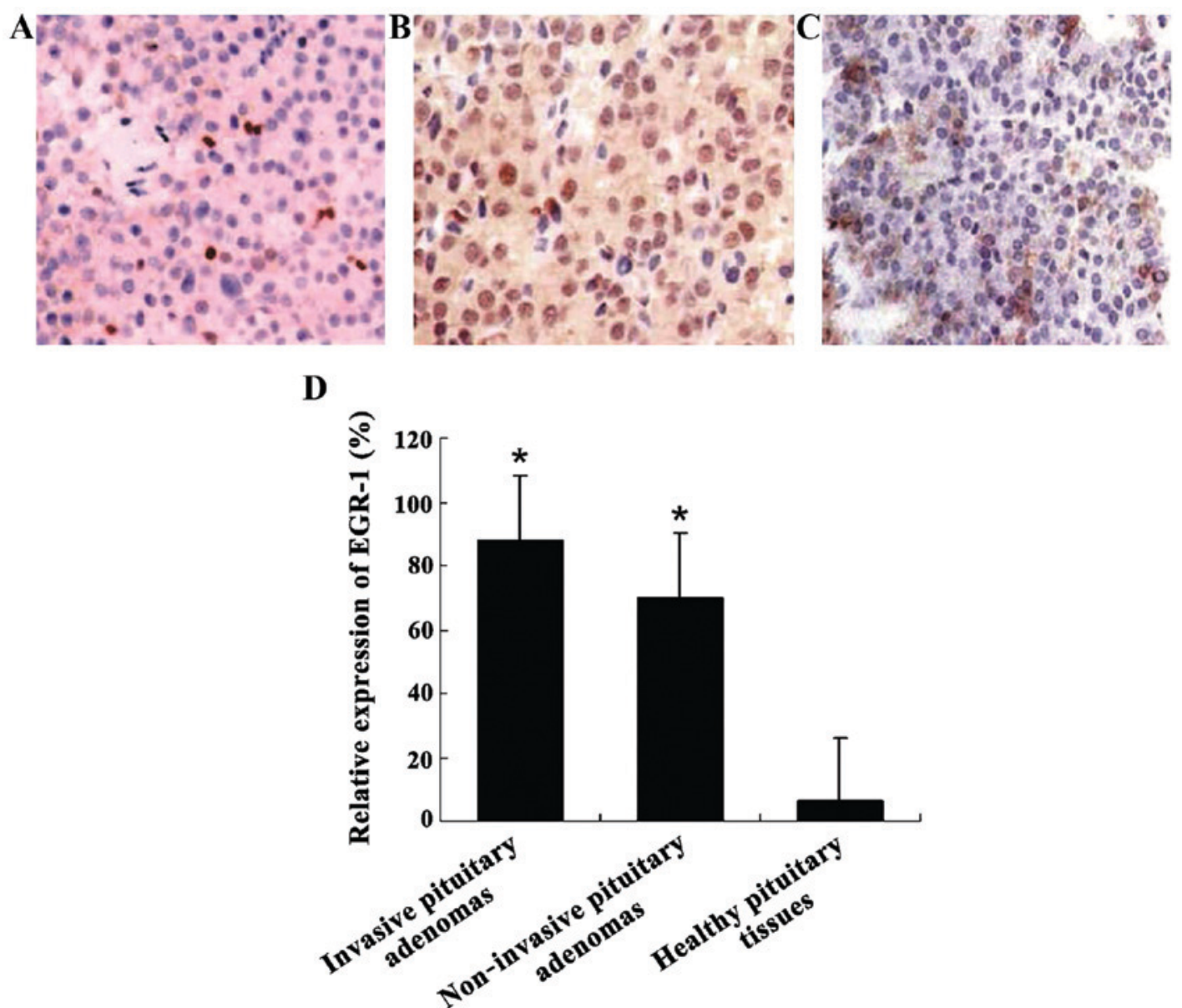

Figure 1. Expression of EGR-1 in different groups was detected by immunohistochemical staining (magnification, $x$ 400). Expression of EGR-1 in (A) healthy pituitary tissues, (B) non-invasive pituitary adenomas, and (C) invasive pituitary adenomas. (D) Statistical results showing significant differences in the expression of EGR-1 in the invasive pituitary tumor group, the non-invasive pituitary tumor group and the healthy pituitary tumor group (compared with healthy pituitary tissues, $\left.{ }^{*} \mathrm{P}<0.05\right)$. EGR-1, early growth response protein 1.

not meet the conditions. Quantitative data were compared and tested by ANOVA. $\mathrm{P}<0.05$ was considered to indicate a statistically significant difference.

\section{Results}

Positive rate of EGR-1 and PTEN in tissues of patients and healthy non-pituitary tumors. We detected the positive rates of EGR-1 and PTEN genes in the tissue samples (Table I). Compared with the EGR-1 positive rate of skin tissue samples of the healthy non-pituitary tumor cases, in elderly patients with pituitary tumor, the EGR-1 and PTEN positive rates had no significant changes. Compared with the healthy pituitary tissue, the PTEN positive rates increased significantly in patients with pituitary tumors. The difference was statistically significant $\left(\chi^{2}=56.7, p<0.05\right)($ Fig. 1). 

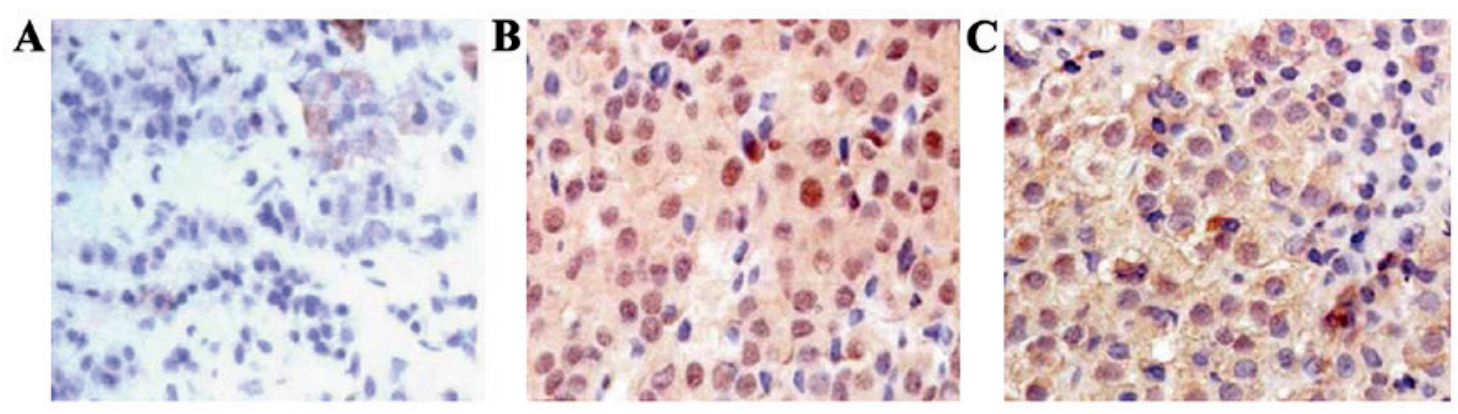

Figure 2. Expression of PTEN in different groups was detected by immunohistochemical staining (magnification x400). (A) Expression of PTEN in healthy pituitary tissues. (B) Expression of EGR-1 in non-invasive pituitary adenomas. (C) Expression of EGR-1 in invasive pituitary adenomas. PTEN, phosphatase and tensin homolog; EGR-1, early growth response protein 1.

Table II. The expression intensity of EGR-1 and PTEN in each group.

\begin{tabular}{lccccr}
\hline Groups & $\begin{array}{c}\text { No. of } \\
\text { cases }\end{array}$ & $\begin{array}{c}\text { EGR-1 expression } \\
\text { intensity }\end{array}$ & $\begin{array}{c}\text { PTEN expression } \\
\text { intensity }\end{array}$ & T-value & P-value \\
\hline Invasive pituitary tumor & 25 & $7.21 \pm 2.3$ & $8.52 \pm 1.7$ & 8.44 & 0.012 \\
Non-invasive pituitary tumor & 10 & $4.62 \pm 1.4$ & $0.27 \pm 0.8$ & & \\
Healthy pituitary tumor tissue & 35 & $0.01 \pm 0.005$ & $0.02 \pm 0.01$ & & \\
\hline
\end{tabular}

EGR-1, early growth response protein 1; PTEN, phosphatase and tensin homolog.

EWxpression intensity of EGR-1 and PTEN in patients and healthy non-pituitary tumors (mean $\pm S D$ ). The average expression intensity of EGR-1 and PTEN protein in pituitary tumors of patients were $7.21 \pm 2.3$ and $4.62 \pm 1.4$, respectively. The expression intensity in normal skin tissues was $8.52 \pm 1.7$ and $0.27 \pm 0.8$, respectively, compared with the healthy non-pituitary tumors. The difference of the expression intensity of EGR-1 and PTEN had statistical significance $(\mathrm{t}=8.44,5.31, \mathrm{p}<0.05)$ (Fig. 2 and Table II).

\section{Discussion}

A pituitary tumor is a common intracranial tumor, whose incidence rate is approximately 1 of 10 million, second only to nerve epidermal tumors and meningiomas. With the development of imaging and endocrine detection technology, the detection rate is increasing annually (12-14). In addition to prolactin, which can be taken in the first trial of the drug sensitivity test for bromocriptine, surgical resection is the first choice for other types of pituitary tumors.

In the diagnosis of pituitary tumors, a pathological diagnosis remains the gold standard, sometimes by means of B-mode ultrasound, computed tomography and other imaging examinations, as well as nasal endoscopic and other methods used to judge the distance of the invasion $(15,16)$. However, these methods are difficult as a substitute for pathological examination. In addition, cancer cells can be present in patients with potential invasive foci. Local invasion foci cannot be detected through general imaging, which brings great challenges to a surgical operation and postoperative treatment.

Previous findings have shown EGR-1 or PTEN can be used as markers for the diagnosis of senile pituitary tumors $(17,18)$.
Of note in evaluating the invasion and prognosis of malignant tumors, in previous studies of PTEN levels in cervical cancer, PTEN was significantly increased in the early stages, but the levels significantly decreased with the increase in the degree of malignancy (19). PTEN competes for substrates with tyrosine kinase as a tumor suppressor. It can play a regulatory role by blocking pituitary growth, mitosis and other factors, but the underlying mechanism is not clear (20). In human solid tumors, the blocking mechanism remains to be confirmed. Based on this, we designed the present study to detect the expression of PTEN in human pituitary adenomas and to determine some of the influencing factors. However, we found that PTEN expression was not high in some patients with pathologically diagnosed pituitary tumors. Therefore, we selected EGR-1 as a common reference detection index.

In previous studies, the EGR-1-encoded protein structure has three repeated zinc finger domains and plays an important role in the interaction between the external signal and the target gene (21). There was a significant increase in many types of malignant tumors, including senile pituitary tumors, but the degree of the increase was not significantly associated with the occurrence and development of tumors and the degree of tumor differentiation $(22,23)$. Through experiments, we found that in patients with non-invasive pituitary tumors, EGR-1 and PTEN levels increased significantly. Conversely, in patients with invasive pituitary tumors, EGR-1 levels increased significantly, albeit the level of PTEN was normal or even low. Taking the possible reasons for our results into account, we believe that in the early stages of pituitary tumor development, due to the impact of various physical and chemical factors, the EGR-1 gene was activated in vivo, further promoting the occurrence of cancer. Concurrently, as the balance lever, the 
tumor suppressor gene PTEN, is also activated. Through the inhibition of tumor pituitary mitosis and in other ways, PTEN inhibits the proliferation of tumor pituitary and distant invasive cells. However, with the progress of the disease, due to the various signaling pathways and the release of inflammatory factors, it results in the activation of the proto-oncogene, which may contain some genes that suppress PTEN signal pathways. This results in the anti-oncogene PTEN weakening the protective effect and promoting tumor invasion. Nevertheless, this hypothesis requires additional theoretical experimentation for possible confirmation.

In conclusion, the present findings have shown that in senile pituitary tumor patients, EGR-1 and PTEN levels were significantly higher than those of healthy individuals, but in patients with distant invasive pituitary tumors, PTEN levels decreased while EGR-1 levels increased. PTEN and EGR-1 can play an important role in the potential invasiveness of pituitary tumors in patients, and this is an area of great significance in guiding clinical surgery and chemotherapy.

\section{References}

1. Beckers A, Aaltonen LA, Daly AF and Karhu A: Familial isolated pituitary adenomas (FIPA) and the pituitary adenoma predisposition due to mutations in the aryl hydrocarbon receptor interacting protein $(A I P)$ gene. Endocr Rev 34: 239-277, 2013

2. Donangelo I, Ren SG, Eigler T, Svendsen C and Melmed S: Sca1 ${ }^{+}$ murine pituitary adenoma cells show tumor-growth advantage. Endocr Relat Cancer 21: 203-216, 2014.

3. Zhu X, Wang Y, Zhao X, Jiang C, Zhang Q, Jiang W, Wang Y, Chen $\mathrm{H}$, Shou X, Zhao Y, et al: Incidence of pituitary apoplexy and its risk factors in Chinese people: a database study of patients with pituitary adenoma. PLoS One 10: e0139088, 2015.

4. Zhou Y, Zhang X and Klibanski A: Genetic and epigenetic mutations of tumor suppressive genes in sporadic pituitary adenoma. Mol Cell Endocrinol 386: 16-33, 2014.

5. Xiao JQ, Liu XH, Hou B, Yao Y, Deng K, Feng M, Xing B, Lian W, Wang RZ and Feng F: Correlations of pituitary tumor transforming gene expression with human pituitary adenomas: a meta-analysis. PLoS One 9: e90396, 2014.

6. Cristina C, Luque GM, Demarchi G, Lopez-Vicchi F, Zubeldia-Brenner L, Perez-Millan MI, Perrone S, Ornstein AM, Lacau-Mengido IM, Berner SI, et al: Angiogenesis in pituitary adenomas: human studies and new mutant mouse models. Int J Endocrinol 2014: 608497, 2014.

7. Waligórska-Stachura J, Andrusiewicz M, Sawicka-Gutaj N, Kubiczak M, Jankowska A, Liebert W, Czarnywojtek A, Waśko R, Blanco-Gangoo AR and Ruchała M: Evaluation of survivin splice variants in pituitary tumors. Pituitary 18: 410-416, 2015.

8. Yang L, Tang J, Chen H, Ge D, Sui T, Que J, Cao X and Ge Y: Taurine reduced epidural fibrosis in rat models after laminectomy via downregulating EGR1. Cell Physiol Biochem 38: 2261-2271, 2016.
9. Fekete $\mathrm{C}$ and Lechan RM: Central regulation of hypothalamicpituitary-thyroid axis under physiological and pathophysiological conditions. Endocr Rev 35: 159-194, 2014.

10. Gaston-Massuet C, Andoniadou CL, Signore M, Jayakody SA, Charolidi N, Kyeyune R, Vernay B, Jacques TS, Taketo MM, Le Tissier $\mathrm{P}$, et al: Increased Wingless $(W n t)$ signaling in pituitary progenitor/stem cells gives rise to pituitary tumors in mice and humans. Proc Natl Acad Sci USA 108: 11482-11487, 2011.

11. Miermeister CP, Petersenn S, Buchfelder M, Fahlbusch R, Lüdecke DK, Hölsken A, Bergmann M, Knappe HU, Hans VH, Flitsch J, et al: Histological criteria for atypical pituitary adenomas - data from the German pituitary adenoma registry suggests modifications. Acta Neuropathol Commun 3: 50, 2015.

12. Heaney AP: Clinical review: pituitary carcinoma: difficult diagnosis and treatment. J Clin Endocrinol Metab 96: 3649-3660, 2011.

13. Mathioudakis N, Sundaresh R, Larsen A, Ruff W, Schiller J, Guerrero-Cázares H, Burger P, Salvatori R and Quiñones-Hinojosa A: Expression of the pituitary stem/progenitor marker GFR $\alpha 2$ in human pituitary adenomas and normal pituitary. Pituitary 18: 31-41, 2015.

14. Lines KE, Stevenson M and Thakker RV: Animal models of pituitary neoplasia. Mol Cell Endocrinol 421: 68-81, 2016.

15. Antoine V, Moret C, Schmitt E, Klein M and Bracard S: MRI imaging of the neural pituitary. Ann Endocrinol (Paris) 69: 181-192, 2008 (In French).

16. Komotar RJ, Starke RM, Raper DM, Anand VK and Schwartz TH: Endoscopic endonasal compared with microscopic transsphenoidal and open transcranial resection of giant pituitary adenomas. Pituitary 15: 150-159, 2012.

17. Birt JA, Nabli H, Stilley JA, Windham EA, Frazier SR and Sharpe-Timms KL: Elevated peritoneal fluid TNF- $\alpha$ incites ovarian early growth response factor 1 expression and downstream protease mediators: a correlation with ovulatory dysfunction in endometriosis. Reprod Sci 20: 514-523, 2013.

18. Palumbo T, Faucz FR, Azevedo M, Xekouki P, Iliopoulos D and Stratakis CA: Functional screen analysis reveals miR-26b and miR-128 as central regulators of pituitary somatomammotrophic tumor growth through activation of the PTEN-AKT pathway. Oncogene 32: 1651-1659, 2013.

19. Tornesello ML, Annunziata C, Buonaguro L, Losito S, Greggi S and Buonaguro FM: TP53 and PIK3CA gene mutations in adenocarcinoma, squamous cell carcinoma and high-grade intraepithelial neoplasia of the cervix. J Transl Med 12: 255,2014

20. Burgucu D, Guney K, Sahinturk D, Ozbudak IH, Ozel D, Ozbilim G and Yavuzer U: Tbx3 represses PTEN and is overexpressed in head and neck squamous cell carcinoma. BMC Cancer 12: 481, 2012.

21. Ozen E, Gozukizil A, Erdal E, Uren A, Bottaro DP and Atabey N: Heparin inhibits hepatocyte growth factor induced motility and invasion of hepatocellular carcinoma cells through early growth response protein 1. PLoS One 7: e42717, 2012.

22. Mellotte G, Maher V, Devitt PG, Shin VY and Leung CP: Minimally invasive surgical oncology: state of the art. Asian Pac J Surg Oncol 1: 101-112, 2015.

23. He J, Yu JJ, Xu Q, Wang L, Zheng JZ, Liu LZ and Jiang BH: Downregulation of ATG14 by EGR1-MIR152 sensitizes ovarian cancer cells to cisplatin-induced apoptosis by inhibiting cyto-protective autophagy. Autophagy 11: 373-384, 2015. 